\title{
Modeling of RF Waves in Free Space Optical Communication System Under Gamma-Gamma Turbulent Channel Effect
}

\author{
Abbas Hamooleh Alipour* and Ali Mir \\ Department of Electrical Engineering, Lorestan University, Khorramabad, Iran \\ *Corresponding Author's Email: alipoor.ab@fe.lu.ac.ir
}

Received: Dec. 20, 2018, Revised: Jun. 21, 2019, Accepted: Aug. 14, 2019, Available Online: Dec. 27, 2019

DOI: 10.29252/ijop.13.2.209

\begin{abstract}
In this paper, an enhancement design of communication system using optical radio frequency (RF) waves in free space optical communication (FSO) system is presented. To our knowledge, it is the first time that the effect of Gamma-Gamma turbulent channel model on the performance of the proposed system is analyzed and simulated. To obtain an optical communication system with good performance and high spectrum efficiency, we proposed two types of optical RF waves including optical single sideband (OSSB) and optical double sideband (ODSB). This strategy that integrated an optical communication system with turbulent channel model can express an accurate model of optical and RF waves propagation in free space. Performance of the system under different regimes, weak, moderate and strong turbulent is studied considering several parameters like max quality factor, bit error rates (BER), and optical signal-to-noise ratio (OSNR). Also, a comparative study between two methods of optical RF waves is presented.
\end{abstract}

KEYWORDS: FSO, OSNR, OSSB, ODSB, Gamma-Gamma turbulent model, BER.

\section{INTRODUCTION}

The technology of free space optical communication (FSO) is offered a system with Gigabit data rate and Ethernet throughput including data, voice and video. Recently, FSO has possessed for several interested features such as high speed transmission data, high bandwidth, low cost and low installation [1-3]. FSO technology for propagation of optical or microwave signals over atmosphere is suffering from variant and random refractive index. This problem decreases the performance of system as well as bit error rate (BER) and optical signal-to-noise ratio (OSNR). In outstretching technology of optical communication system, several systems use the radio-over-fiber (ROF) technology. The main purpose of this method is integration with other networks and low dispersion [4-5]. But, integration the radio frequency (RF) wave with FSO system is hardly affected by the atmosphere turbulent that is one of the advantage of radio over free space communication [6]. Also, the propagated optical beam through the atmosphere can be scattered and absorbed by different atmosphere condition. The interaction between optical or RF waves with turbulence can be degraded the phase and amplitude of received waves. In order to model the turbulent channel of FSO systems several methods including long normal, negative exponential and Gamma-Gamma channel model are presented [7-9]. In [10], several types of optical RF waves including optical single sideband (OSSB), optical double sideband (ODSB) and optical-carrier-suppression (OCS) are proposed. These methods can be considered using direct and external modulation. Direct modulation with laser source is simpler than external modulation, but it has drawbacks of limited transmission of data rate and limited bandwidth. A radio over free space optical communication system with different channel model is presented in [11]. The system only analyzed the optical spectrum of waves before and after the free space channel. In [12], BER 
performance of binary phase shift keying (BPSK) modulation in FSO link based on long-normal turbulent channel model is simulated.

In this paper, a new optical communication system with two types of optical RF waves, OSSB and ODSB, by considering the effect of Gamma-Gamma turbulent channel model is introduced. In weak and moderate regimes, two types of optical RF waves have good performance, but in strong regime the ODSB waves has a better operation rather than OSSB wave.

The rest of the paper is organized as follows: in section 2 and 3 the Gamma-Gamma turbulent model of free space channel and generation of optical RF waves are explained. Proposed system and simulation results are expressed in section 4. Finally we conclude the paper in section 5 .

\section{GAMMA-GAMMA TURBULENT Channel Model}

The performance of FSO link is affected with atmosphere turbulence which causes some changes in refractive index. The model of turbulent channel is very complex and depended on the earth's surface that can be studied in weak, moderate and strong regimes. One of the turbulent channel models is Gamma-Gamma model which is operated based on modulation processes. The GammaGamma turbulent channel model can be modeled by received irradiance $I[13]$ :

$$
I=I_{x} I y
$$

where $I_{x}$ and $I_{y}$ are large and small scale of turbulent eddies, respectively. Also, the probability density function (PDF) of received irradiance distribution function is given as [13]:

$P(I)=\frac{2(\alpha \beta)^{(\alpha+\beta) / 2}}{\Gamma(\alpha)+\Gamma(\beta)} I^{(\alpha+\beta) / 2} K_{\alpha-\beta}(2 \sqrt{\alpha \beta I})$

where $K_{n}(0)$ is the modified Bessel function of second kind of order $n . \alpha$ and $\beta$ are the parameters of Gamma-Gamma distribution related to large-scale and small-scale scintillation, respectively, and $\Gamma(0)$ is the Gamma function assuming that the optical radiation is a plan wave. Also, $\alpha$ and $\beta$ are given by [13]:

$$
\begin{aligned}
& \alpha=\left\{\exp \left[\frac{0.4 \sigma_{R}^{2}}{\left(1+1.11 \sigma_{R}^{2}\right)^{7 / 6}}\right]\right\}^{-1} \\
& \beta=\left\{\exp \left[\frac{0.51 \sigma_{R}^{2}}{\left(1+0.69 \sigma_{R}^{12 / 5}\right)^{5 / 6}}\right]^{-1}\right\}^{-1}
\end{aligned}
$$

where $\sigma_{R}^{2}$ is the long irradiance variance and given as [14]:

$$
\sigma_{R}^{2}=1.23 c_{n}^{2} k^{7 / 6} L^{11 / 6}
$$

where $L$ is the link path, $k=2 \pi / \lambda$, is the wave number and $c_{n}^{2}$ is the refractive index structure. The PDF of $P(I)$ versus irradiance, $I$, for different regime and $\sigma_{R}^{2}$ is shown in Fig. 1.

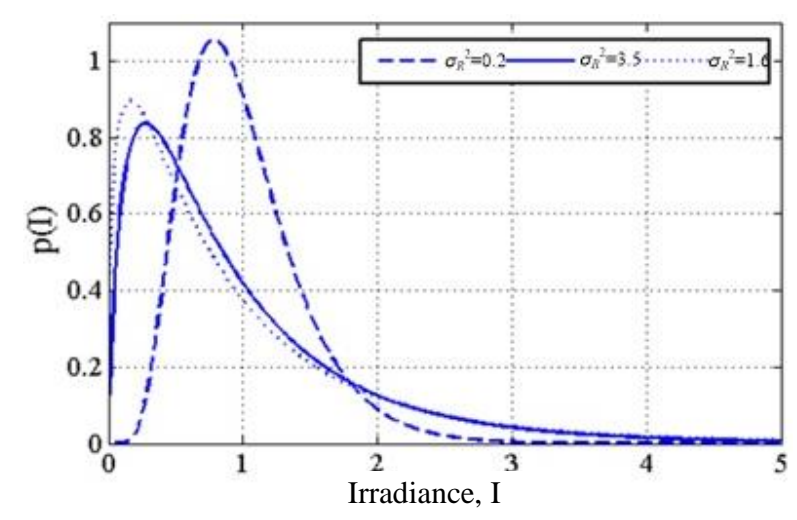

Fig. 1. PDF of the proposed system versus the irradiance.

Table 1. Different parameters, used in the GammaGamma model

\begin{tabular}{cccc}
\hline \hline parameter & weak & moderate & strong \\
\hline \hline$A$ & 11.651 & 4.026 & 4.225 \\
$B$ & 10.153 & 1.976 & 1.484 \\
$\sigma_{R}^{2}$ & 0.2 & 1.6 & 3.5 \\
$c_{n}^{2}$ & $7.5 \times 10^{-17}$ & $3.6 \times 10^{-15}$ & $1.7 \times 10^{-13}$ \\
\hline \hline
\end{tabular}

Table 1 summarized the different parameters of the Gamma-Gamma turbulent channel 
model in different regimes such as weak, moderate and strong.

\section{Generation OF OPTICAL RF WAVES}

To generate the optical RF waves, two methods of direct and external modulation can be used. Direct modulation unlike external modulation has several restrictions such as low data rate (less than $1 \mathrm{Gbps}$ ) and low modulation bandwidth of laser source. Also, one of the advantage of external modulation is low nonlinear effects of modulator. Based on mentioned reasons, we proposed the external modulation for our presented system. Figure 2 shows the configuration of ODSB and OSSB scheme. Mach-Zehender modulator (MZM) was biased with RF input signal and laser used as optical carrier wave [14].

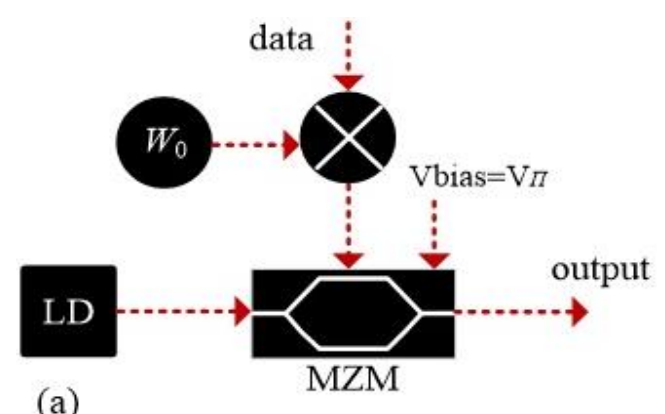

(a)

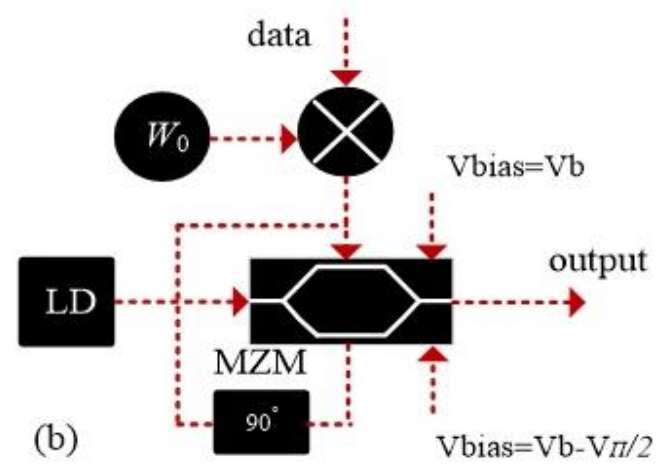

Fig. 2. Configuration of the proposed optical RF waves, (a) ODSB wave, (b) OSSB wave.

Figure 3 shows the optical spectrum output of MZM for proposed optical RF waves. It is clear that ODSB wave has higher power than OSSB wave. So, in the next section we try to demonstrate that the performance of ODSB wave is better than OSSB wave in the proposed system.

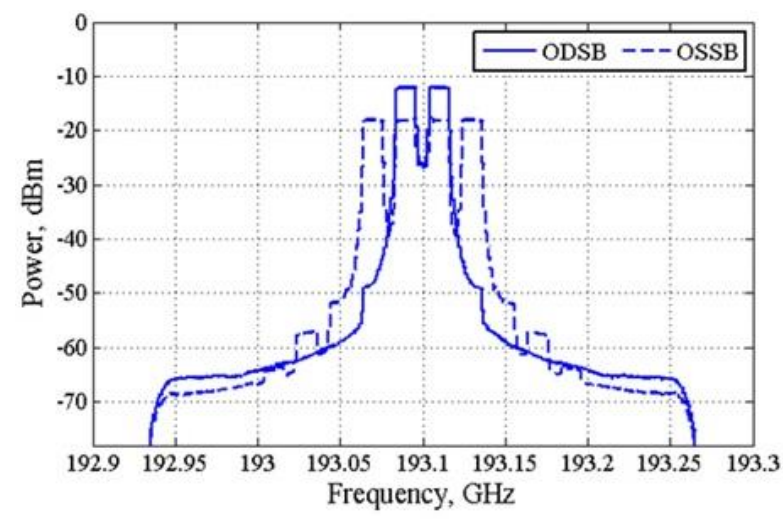

Fig. 3. Optical output spectra of MZM for ODSB and OSSB waves.

\section{IV.PROPOSED SYSTEM AND SiMULATION RESULTS}

The block diagram of the proposed system is shown in Fig. 4. The presented system consists of optical RF waves that are prepared in section III. Optical RF waves have been transmitted through the atmosphere channel and detected by the receiver unit. The transmitted unit uses a single wavelength continuous-wave (CW) laser at $193.1 \mathrm{THz}$ consider into account ODSB format. Figure 5 shows the block diagram of the receiver unit that consist of optical filter (OF), optical amplifier (OA), PIN photodiode (PIN PD), amplitude modulation (AM) and analyzer. In Table II, the setup parameters of the proposed system are shown. The link distance of the proposed system is $1500 \mathrm{~m}$ that operates at 10 Gbps rate.

Table 2. Maximum quality factor and minimum BER at different regimes

\begin{tabular}{|c|c|c|c|c|}
\hline \multirow{2}{*}{$\begin{array}{l}\text { Gamma- } \\
\text { Gamma } \\
\text { condition }\end{array}$} & \multicolumn{2}{|c|}{ Q } & \multicolumn{2}{|c|}{ BER } \\
\hline & OSSB & ODSB & OSSB & ODSB \\
\hline Weak & 12.108 & 15.15 & $5.37 \times 10^{-34}$ & $2.93 \times 10^{-52}$ \\
\hline Moderate & 8.83 & 12.12 & $2.36 \times 10^{-21}$ & $3.37 \times 10^{-34}$ \\
\hline Strong & 2.82 & 4.19 & $2.2 \times 10^{-3}$ & $1.31 \times 10^{-5}$ \\
\hline
\end{tabular}

Turbulent channel model and optical RF waves are considered simultaneously for simulation of the proposed system, at clear weather atmosphere with $0.18 \mathrm{~dB}$ attenuation. Eye diagram and max quality factor of the proposed system, versus time, are shown in Fig. 6, for two ODSB and OSSB schemes at $10 \mathrm{Gbps}$ rate. It is obvious that ODSB wave 
has better performance and max quality in different regimes. Table III shows the results of the presented system including max quality factor, min BER and OSNR at different regimes.

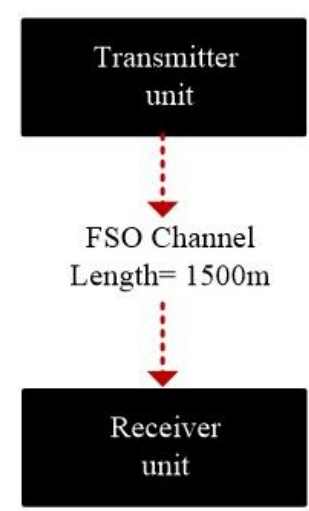

Fig. 4. Overall block diagram of the proposed system.

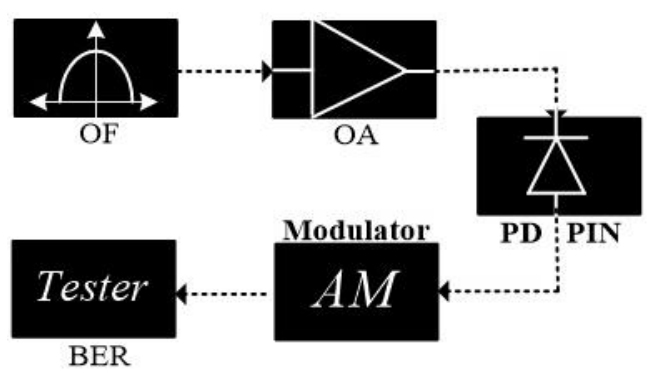

Fig. 5. The block diagram of the receiver (OA: optical amplifier, OF: optical filter, PD: photodetector, AM: amplitude modulation).

A comparison between min BER, max quality factor and OSNR for two methods shows that ODSB waves have better performance than OSSB waves at different regimes. For example results at moderate regime ODSB shows 12.12 max quality factor, $3.37 \times 10^{-34}$ min BER and 46.85 OSNR, also for OSSB at this regime max quality, min BER and OSNR are 8.83, $2.36 \times 10^{-21}$ and 46.6, respectively. Figure 7 and Fig. 8 show OSNR, and Min log BER of the proposed system versus input powers for OSSB and ODSB schemes. It is illustrated that by enhancement of the input power OSNR of the system will be improved and the Min log BER is reduced in two proposed methods. However, increasing the value of input power can increase insertion loss of the system. Also the results show that at weak regime the variation is less than moderate and strong turbulent channel model.
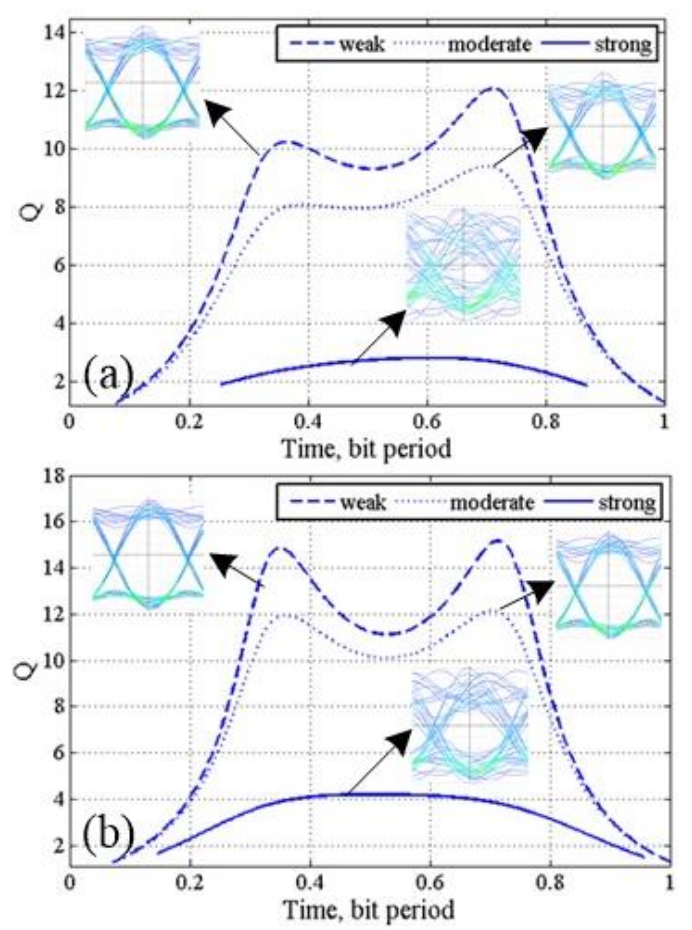

Fig. 6. The eye diagram and quality factor, $Q$ of (a) OSSB and (b) OSDB at 10 Gbps.

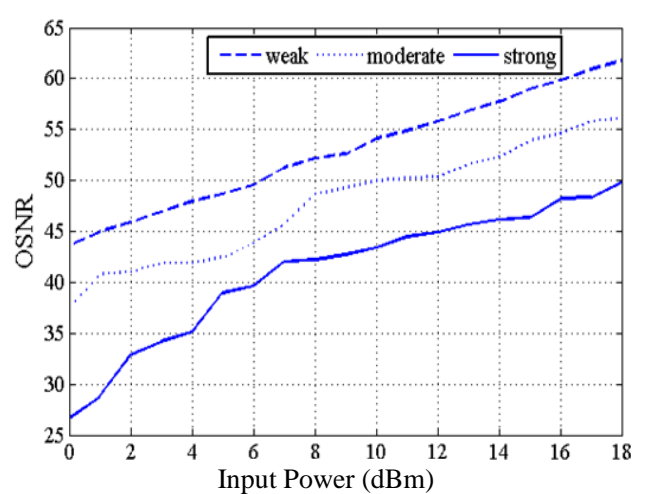

(a)

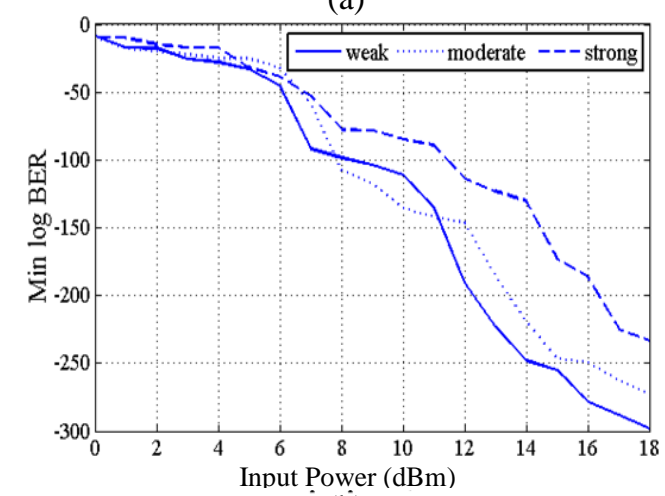

(b)

Fig. 7. (a) OSNR, and (b) Min log BER in OSSB wave versus input power of the system. 
In our future works, we try to design and analyze the presented system with Dense Wavelength Division Multiplexing (DWDM) system and different channel model to achieve next generation networks.

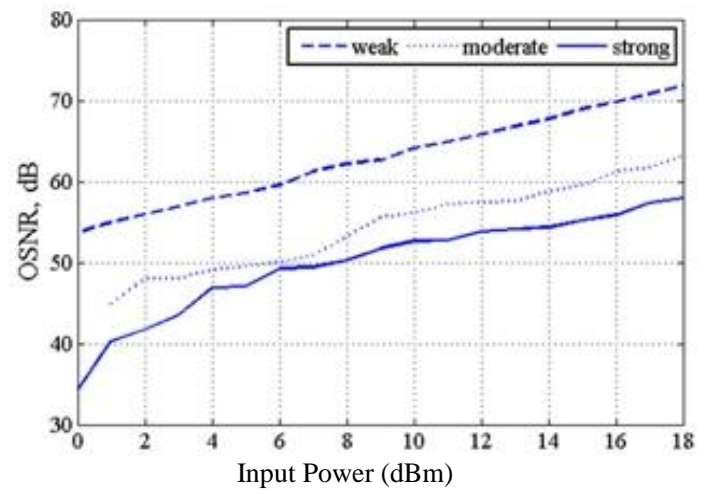

(a)

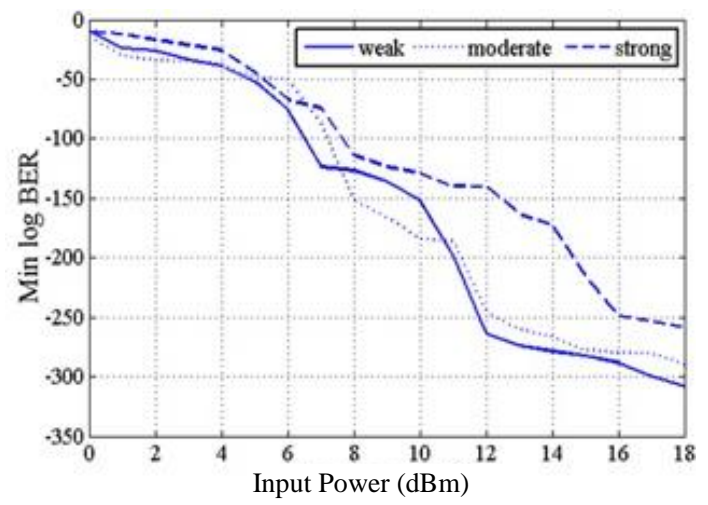

(b)

Fig. 8. (a) OSNR, (b) Min log BER in ODSB wave versus input power of the system.

Table 3. The maximum quality factor and minimum BER for different atmospheric conditions

\begin{tabular}{ccccccc}
\hline \hline \multirow{2}{*}{$\begin{array}{c}\text { Gamma-Gamma } \\
\text { condition }\end{array}$} & OSSB & ODSB & OSSB & ODSB & OSSB & ODSB \\
\hline \hline Weak & 12.108 & 15.15 & $5.37 \times 10^{-34}$ & $2.93 \times 10^{-52}$ & 47.35 & 48.71 \\
Moderate & 8.83 & 12.12 & $2.36 \times 10^{-21}$ & $3.37 \times 10^{-34}$ & 46.6 & 46.85 \\
Strong & 2.82 & 4.19 & $2.2 \times 10^{-3}$ & $1.31 \times 10^{-5}$ & 39.59 & 39.61 \\
\hline \hline
\end{tabular}

\section{Conclusion}

In this paper, we have proposed and analyzed a new design of optical microwave over free space communication system by taking into account Gamma-Gamma turbulent channel model and attenuation loss. The proposed system is simulated and enhanced to obtain min BER and OSNR, and max quality factor at 10 Gbps through a $1500 \mathrm{~m}$ distance link with two methods of optical RF waves, OSSB and ODSB. From the simulation results we understand that ODSB wave has better performance for proposed system rather than OSSB wave at different regimes. A system with these features is suitable for using in the wireless communication systems.

\section{REFERENCES}

[1] D. Killinger, "Free space optics for laser communication through the air," Optics and Photonics News, Vol. 13, pp. 36-42, 2002.

[2] K. Wakafuji and T. Ohtsuki, "Performance analysis of atmospheric optical subcarriermultiplexing systems and atmospheric optical subcarrier-Modulated code-division multiplexing systems," J. Lightw. Technol. Vol. 23, pp. 1676-1682, 2005.

[3] A. Alipour, A. Mir, and A. Sheikhi, "Ultra high capacity inter-satellite optical wireless communication system using different optimized modulation formats," Optik- Inter. J. Light Electron Opt. Vol. 127, pp. 81358143, 2016.

[4] J. Bohata, S. Zvanovec, T. Korinek, M. Mansour Abadi, and Z. Ghassemlooy, "Characterization of dual-polarization LTE radio over a free-space optical turbulence channel," Appl. Opt. Vol. 54, pp. 7082-7087, 2015.

[5] R. Montgomery and R. DeSalvo, "A novel technique for double sideband suppressed carrier modulation of optical fields," IEEE Photon. Technol. Lett. Vol. 7, pp. 434- 436, 1995.

[6] Z. Ghassemlooy, W.O. Popoola, and E. Leitgeb "Free-Space Optical Communication Using Subcarrier Modulation in GammaGamma Atmospheric Turbulence," 9th International Conference on Transparent Optical Networks, Vol. 3, pp. 156-160, 2007.

[7] A.M. Mbah, J.G. Walker, and A.J. Phillips, "Performance evaluation of turbulence 
accentuated inter channel crosstalk for hybrid fiber and free-space optical wavelength division-multiplexing systems using digital pulse-position modulation," IET Optoelectron., Vol. 10, pp. 11-20, 2016

[8] Z. Ghassemlooy, W. Popoola, and S. Rajbhandari, Optical wireless communications: system and channel modelling with Matlab, CRC press, 2019.

[9] G-K. Chang, Y-T. Hsueh, and S-H. Fan, "Advances in 1-100 GHz Microwave Photonics: All-Band Optical Wireless Access Networks Using Radio over Fiber Technologies," Opt. Fib. Telecommun. pp. 873-889, 2013.

[10] A.H. Alipour, A. Mir, and A. Sheikh, "UltraHigh Capacity DWDM System using Different Intensity Modulation Formats,' Majlesi J. Elec. Eng. Vol. 12, pp. 95-101, 2018.

[11]K. Prabu, R. Rajendran, and D.S. Kumar, "Spectrum analysis of radio over free space optical communications systems through different channel models," Optik- Inter. J. Light Electron Opt., Vol. 126, pp. 1142-1145, 2015.

[12]X. Tang, S. Rajbhandari, W.O. Popoola, Z. Ghassemlooy, S.S. Muhammad, E. Leitgeb, and G. Kandus, "Performance of BPSK Subcarrier Intensity Modulation Free-Space Optical Communications using a Log-normal Atmospheric Turbulence Model," Symposium on Photonics and Optoelectronic (SOPO), pp. 1-4, 2010.

[13]G.K. Varotsos, A.N. Stassinakis, H.E. Nistazakis, A.D. Tsigopoulos, and K.P. Peppas, "Probability of fade estimation for FSO links with time dispersion and turbulence modeled with the gamma-gamma or the I-K distribution," Optik- Inter. J. Light Electron Opt. Vol. 125, pp. 7191-7197, 2014.

[14] M. Uysal, J. Li, and M. Yu, "Error rate performance analysis of coded free-space optical links over gamma-gamma atmospheric turbulence channels," IEEE Trans. Wirel. Commun, Vol. 5, pp. 12291233, 2006.

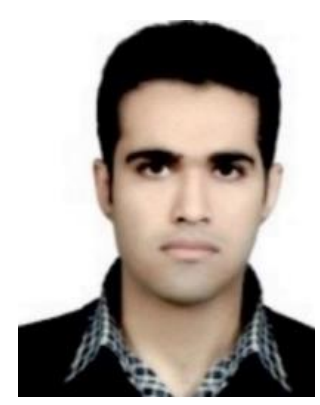

Abbas Hamooleh Alipour received the B.E. degree in Electronic Engineering from Islamic Azad University, Shushtar Branch in 2012, and M.S. degree from Lorestan University in 2016. He is currently an electronic Ph.D. candidate at Lorestan University. His interests fields are optical communication systems and design of optical networks.

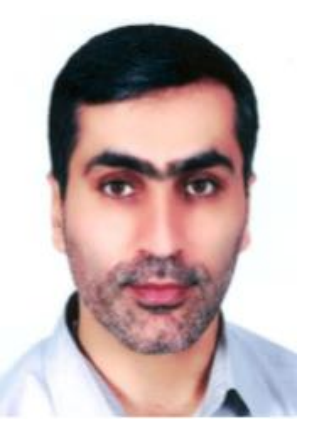

Ali Mir received B.S. degree in Electrical Engineering from Isfahan University of Technology, Isfahan, Iran, in 1997, and M.S. and Ph.D. degree in Electrical Engineering from Tarbiat Modares University in 1999 and 2009, respectively, Tehran, Iran. Now he is an associate professor of electronics at Lorestan University, Khorram-Abad, Iran. His current research activities include Quantum Devices, Photonic Crystals, Optoelectronics and Plasmonics. 